\title{
GEOPHYSICAL RESPONSE OF THE MISSION CREEK FAULT NEAR DESERT HOT SPRINGS, CALIFORNIA
}

\author{
Benjamin J. Drenth, Douglas Moore, Charles. T. Young, Michigan Technological University, \\ Houghton, Michigan, USA
}

\begin{abstract}
Geophysical surveys crossed the Mission Creek Fault, a branch of the San Andreas Fault system near Desert Hot Springs, California. The fault is situated in the alluvial Coachella Valley, and is a major barrier to groundwater flow. The fault trace is marked sporadically by oases and vegetation, and is associated with hot springs and hot water wells. Resistivity, VLF, VLFresistivity, and horizontal-loop electromagnetics (HLEM) have been employed to visualize the subsurface near the fault, and image the fault gouge. The main geophysical response is due to the water table which is deeper on the southwest side of the fault, according to observations in nearby wells. We believe that the geophysical response of the fault is distinct enough to allow its delineation in other locations where not exposed and/or mapped. The measurements were carried out as an undergraduate field trip and class project.
\end{abstract}

\section{Introduction}

Desert Hot Springs is located within the Coachella Valley in southern California. The Coachella Valley is bordered by mountains on the North, South, and West and two branches of the San Andreas Fault system run through the valley. The valley contains thick sand and gravel sedimentary sequences eroded from the surrounding mountains. The sedimentary section is several thousand feet thick and thickens from north to south. North-west south-east striking faults within the valley, including the Mission Creek Fault, act as barriers to the flow of water, which is from northeast to southwest. Thus, the groundwater table is significantly higher on the north-east side of the fault, 1 . The hydrogeology of the basin has been the subject of MS theses 2 and 3.

In some places, the location of the fault is well known because there is a scarp, lines of hot springs and vegetation due to near-surface water. We have conducted geophysical measurements where the location of the fault is well known to establish the usefulness of electrical and electro magnetic geophysical measurements with the intent of future work to locate the fault where there is no surface expression.

The geophysical model for this setting is simple; dry sediment is resistive, saturated sediment is conductive, and there is a difference in water level on each side of the fault. Thus we expect a layered model with a step discontinuity. 


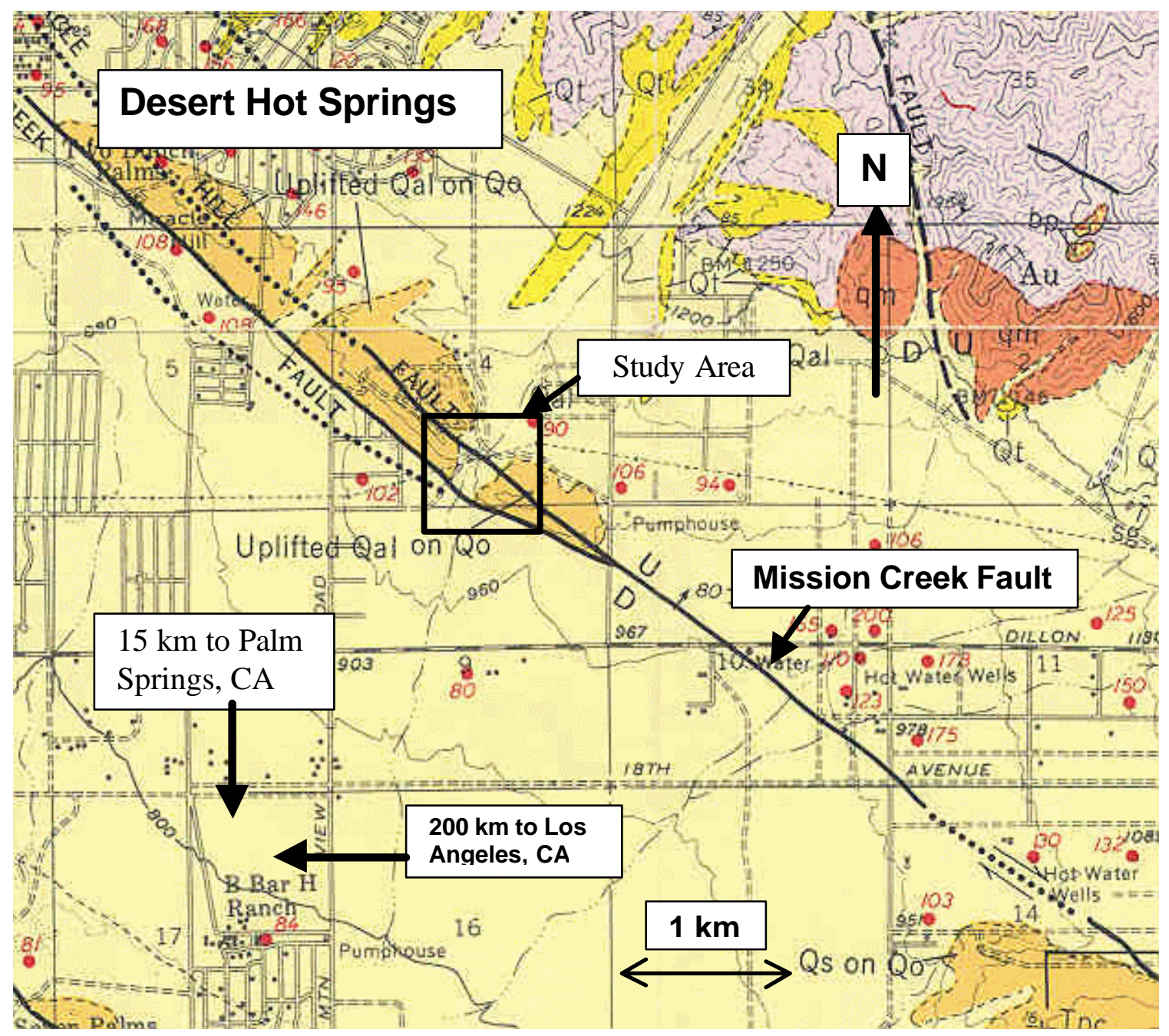




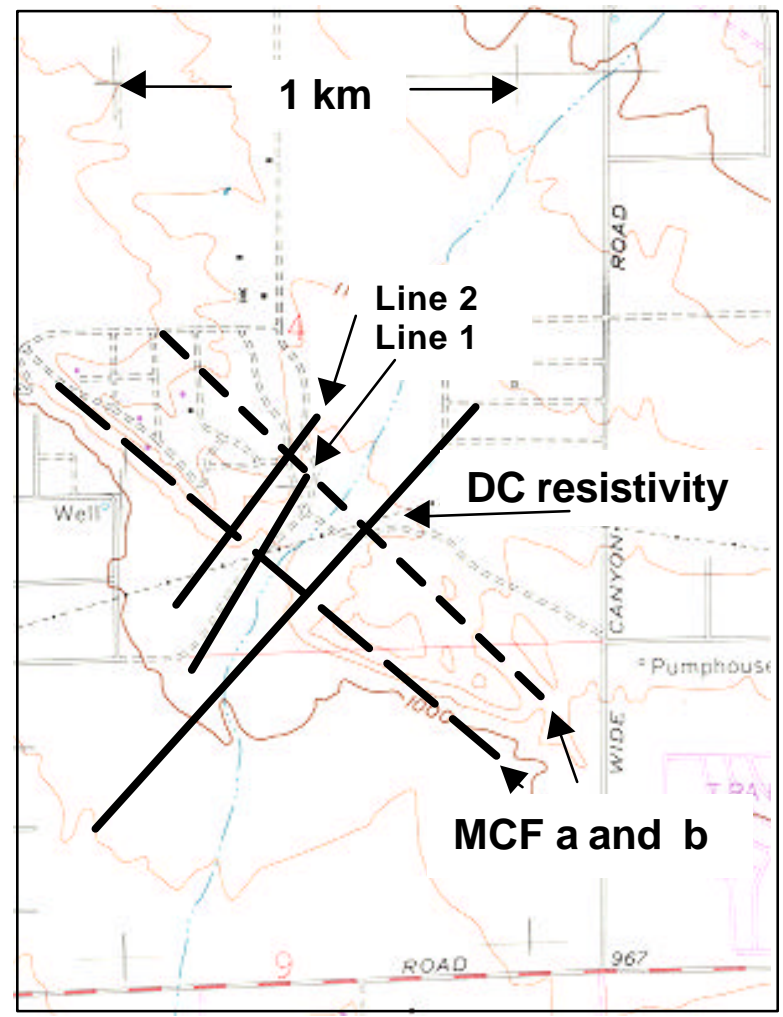

Figure 1. a. (upper) Survey location on geologic map.

b. (lower) Survey lines on detailed topographic map. Traces of the two branches of the Mision Creek Fault are indicated as MCF a and b.

\section{Electrical Resistivity}

\section{Methods}

A series of 13 one-dimensional Schlumberger soundings were made east of Desert Hot Springs, with the current electrode half spacings (AB/2) ranging from 3 to 200 meters (Jimmy F. Diehl personal communication and 3. Layered earth models using Zhody's algorithm, 4, were projected onto a line perpendicular to the fault.

\section{HLEM}

Data were collected along two lines using an Apex Max-Min I HLEM instrument. The transmitter-receiver spacing was 100 meters and the station spacing was 50 meters. In-phase and quadrature data were obtained at 110, 220, 440, 880, 1760, 3520, 7040, and $14080 \mathrm{~Hz}$. This transmitter-receiver spacing provides maximum sensitivity to a conductive layer at about 40 meters depth and is sensitive to layers from zero to about 150 meters depth, 5 .

Layered models for each station were obtained with the program EMIX-MM, 6. The starting model consisted of two layers with Layer 1 resistivity of $1000 \Omega$-m, Layer 1 thickness of 50 meters, and Layer 2 resistivity of $50 \Omega$-m. Modeling was iterated until variation between iterations were minimal and the results fell within five to ten percent error. Data not fitting 
these criteria were either modeled as a three-layer case or model parameters were manually altered within the 2-layer case in order to achieve results consistent with adjacent stations.

\section{$V L F$}

A tilt angle survey was conducted using a Geonics EM-16 with station spacings of 20 meters for Line 1 and 25 meters for Line 2. The direction to the VLF transmitter was approximately northwest; for measurements, the operator faced northeast, so that south tilts were recorded as positive tilt. Tilt angle and quadrature were recorded at each station. For interpretation, the data were filtered to obtain the equivalent subsurface current density, 7, at a depth equal to the station spacing. The filter can be applied to decimated data to create a vertical cross-section of equivalent current density. The filter is blind to a horizontal layer of current of infinite horizontal extent; it detects only the edge of such a layer.

\section{$V L F-R$}

VLF resistivity was measured at the same stations as tilt angle using the EM-16R attachment to the EM-16. The method uses ratio of the horizontal electric and magnetic fields to calculate the apparent resistivity. The instrument reads directly in apparent resistivity in ohmmeters and impedance phase in degrees, 8. The phase angle indicates resistivity changes with depth: a phase of 45 degrees indicates no change in resistivity with depth, a phase less than 45 degrees indicates an increase in resistivity with depth, and a phase of greater than 45 degrees indicates a decrease in resistivity with depth. The apparent resistivity and the phase angle data were modeled using two-layer modeling charts provided with the instrument. These curves require an assumption of a first layer resistivity; the charts may then be used to determine the thickness of the first layer and the resistivity of the bottom half-space. We used charts for first layer resistivities of $1000 \Omega$-m and $3000 \Omega$-m (among others); which are similar to the observed dc resistivities of the shallowest subsurface; two different cross sections were prepared, one for each first layer resistivity assumption.

\section{Results}

\section{Electrical Resistivity}

The resistivities determined from Schlumberger soundings are presented in Figure 2. Please note that the resistivity line covers about 1400 meters, and that the electromagnetic measurements cover only the central portion. The figure indicates generally decreasing resistivity with depth. The profile shows a $300 \Omega$-m layer (green band in the lower figure) located at a depth of roughly 65 meters on the south end of the line, and between 18-30 meters on the north side. This low resistivity layer is interpreted to correspond to saturated sediments, and agrees reasonably well with the depth to the water table observed in nearby wells. On the northwest end of the survey line, high resistivity values are present at anomalously greater depths, possible due to presence of the branch of the fault shown Figure 1. 


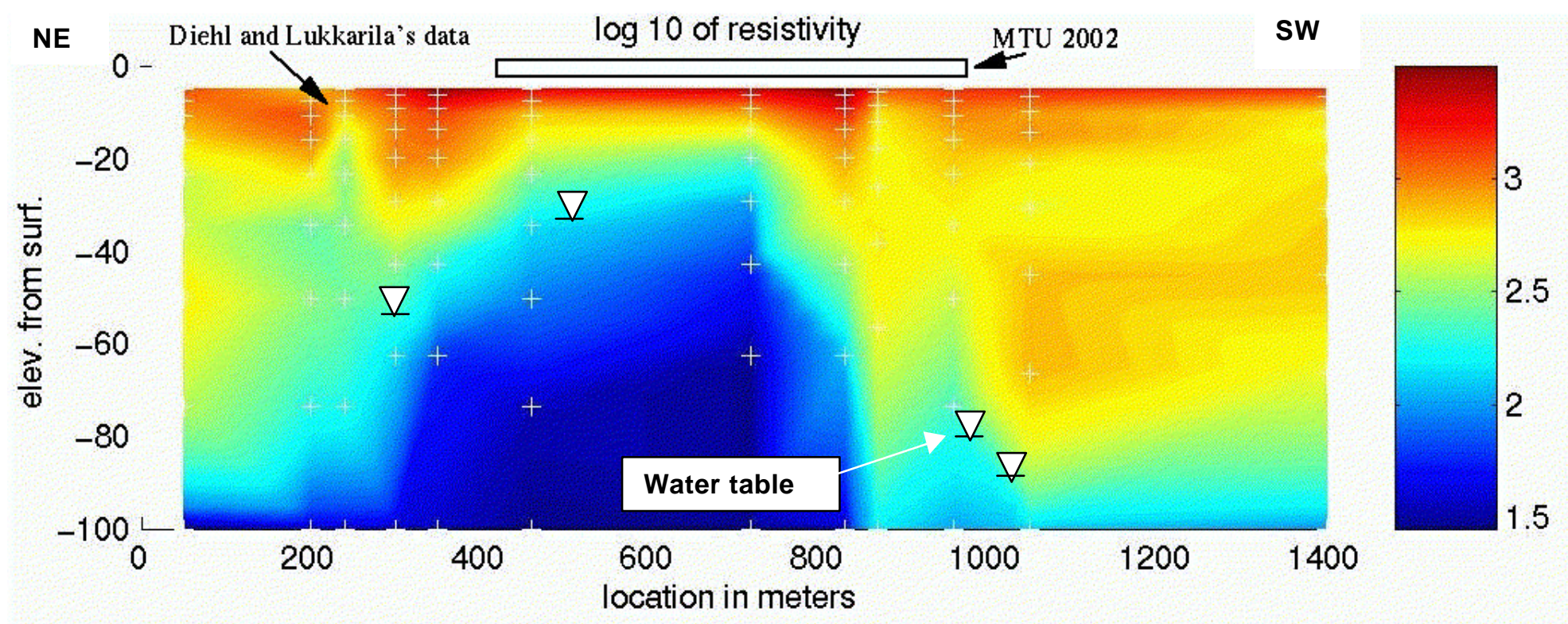

Figure 2: Resistivity cross-section. The color scale represents the logarithm to the base 10 of the resistivity in ohm-m. Northeast is to the left. The white crosses indicate the location of the resistivity stations, and the locations of the top of each interpreted layer. The figure shows a thin, high resistivity layer near the surface, with a moderate resistivity layer and a deep, low resistivity layer at depth. Depths to the water table from nearby wells are shown as triangles

The HLEM data are presented in Figure 3 as cross sections with the highest frequency at the top of the section. Higher frequency data will have shallower penetration due to the skin depth effect. The cross-sections show that there is a significant lateral change in the upper layers at about 400 meters along the line, especially as shown by the quadrature (OOP) components for both lines. This change is interpreted to be due to differing hydrogeologic conditions near the surface, since the groundwater table is much deeper on the south side of the fault. Also, the cross-sections show that this effect is mainly nonexistent at greater depths, indicating that the hydrogeologic changes are greatest near the surface. The cross-sections of the in-phase measurements show subtle discontinuities near the changes in the quadrature measurements, possibly indicating the location of the fault. 

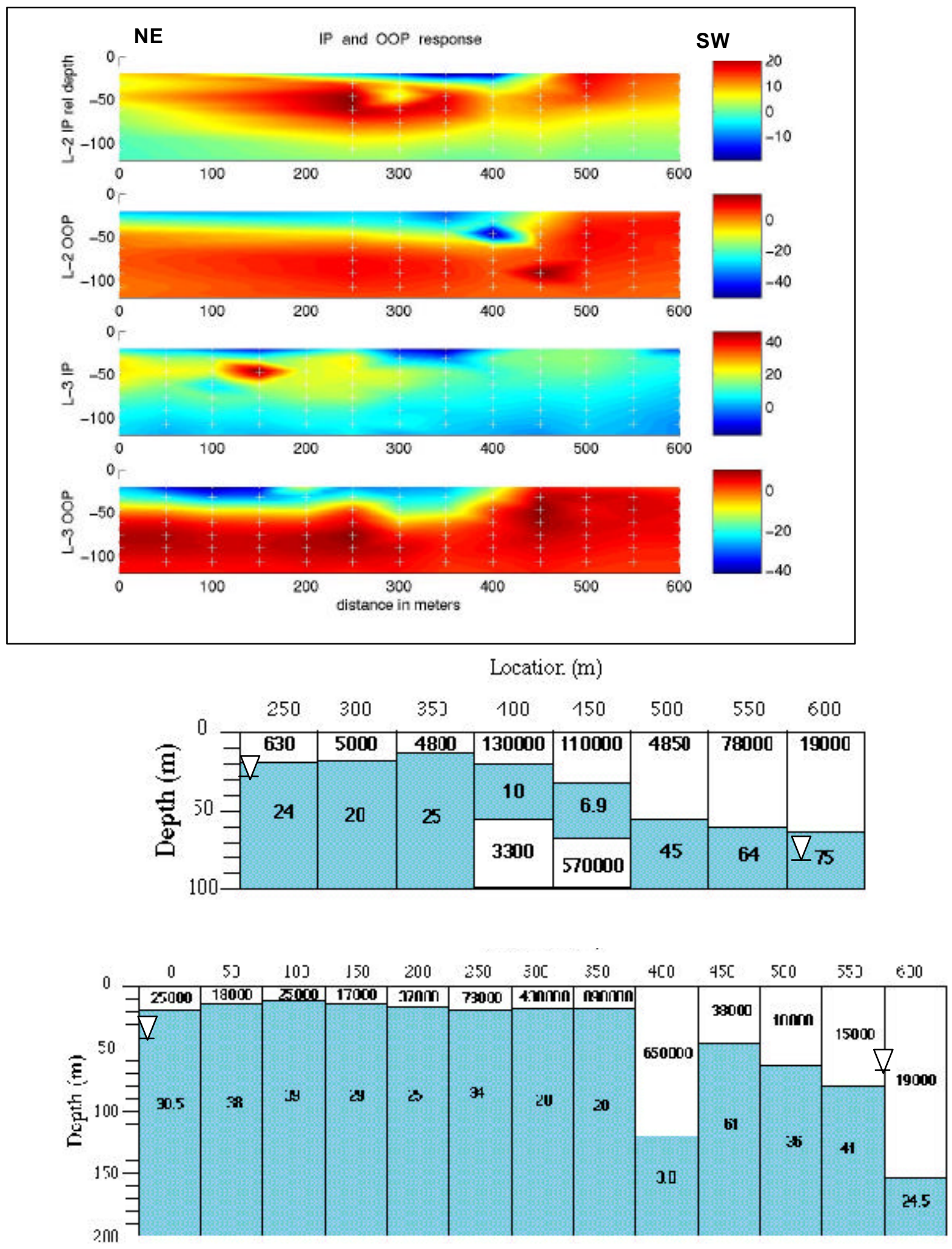

Figure 3: a. (top) In-phase and out-of-phase response for the HLEM survey. Units are percent of the primary field. High frequencies are plotted at the top and low frequencies at the bottom. b. Layered resistivity models for line 2 and line 3. Resistivity in ohm-meters is given for each station. Northeast is to the left. 
Cross sections of HLEM models (Figure 2b) indicate a change in the depth to the conductive layer from depth of 20 meters or less the north end of the lines to 45 or more meters on the south portion of the survey lines.
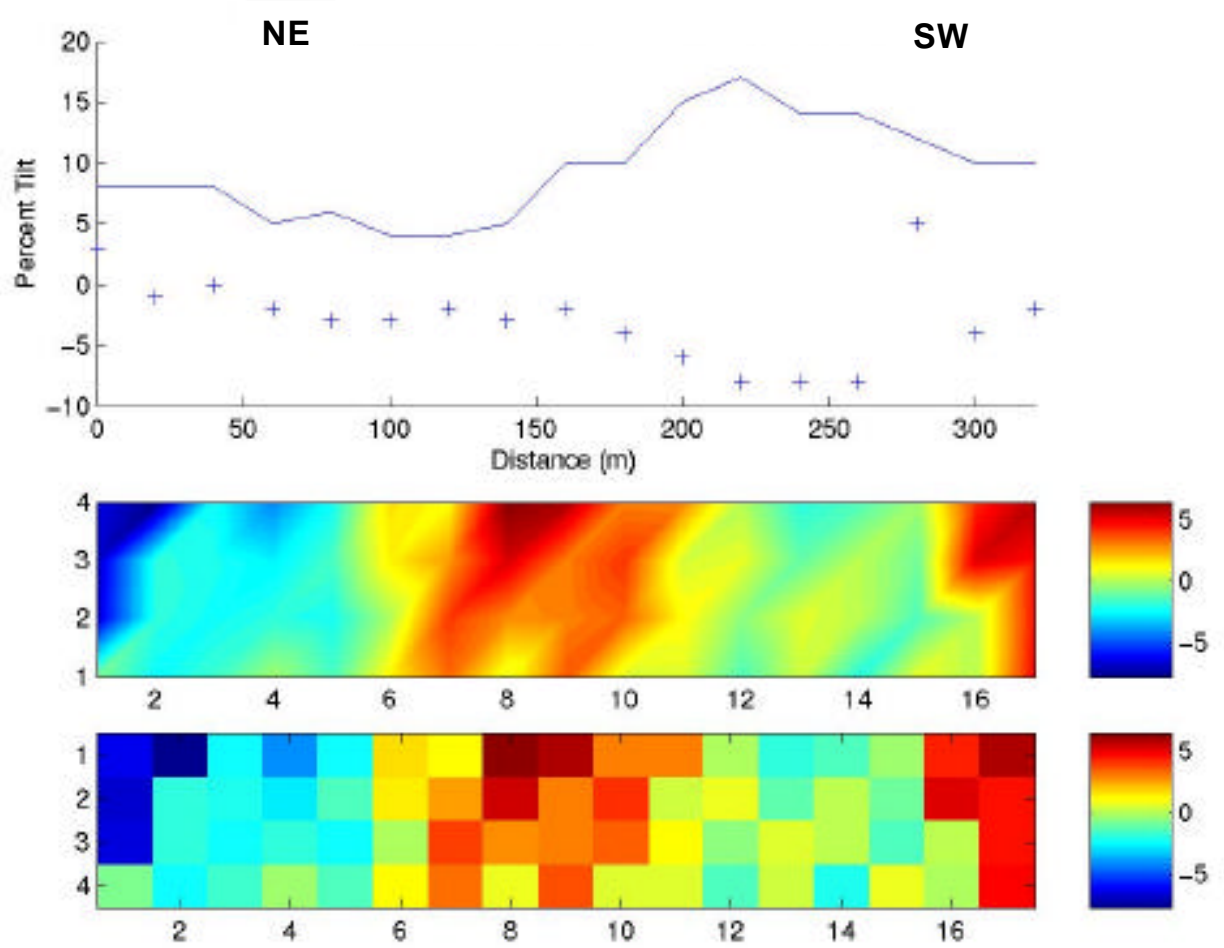

Figure 4: a) Percent tilt (solid line) and quadrature for VLF tilt angle survey for Line 2. b) Equivalent current cross section shown as both smoothed and blocks of current on line 2 . The units are simply the output of the filter. Red is the highest current density, and blue (negative) values are a computational artifact and should be ignored.

One of the lines of VLF tilt angle data were not used because they showed a large tilt anomaly directly under and adjacent to a high tension power line traversing the site. The other line yielded realistic data, which shows an increase followed by decrease in tilt angle centered about 220 meters. The computed current densities in Figure 4 indicate two areas of concentrated equivalent current, occurring at about 150 and 320 meters. The areas of high current flow at 320 and zero meters are most likely edge effects caused by the termination of the data. Two interpretations of the current concentration in the center of the survey include a large conductor or a step discontinuity going from conductive area to a less conductive area. The second explanation is more plausible due to the lack of a crossover in the VLF data that would be expected with a thin conductor. 


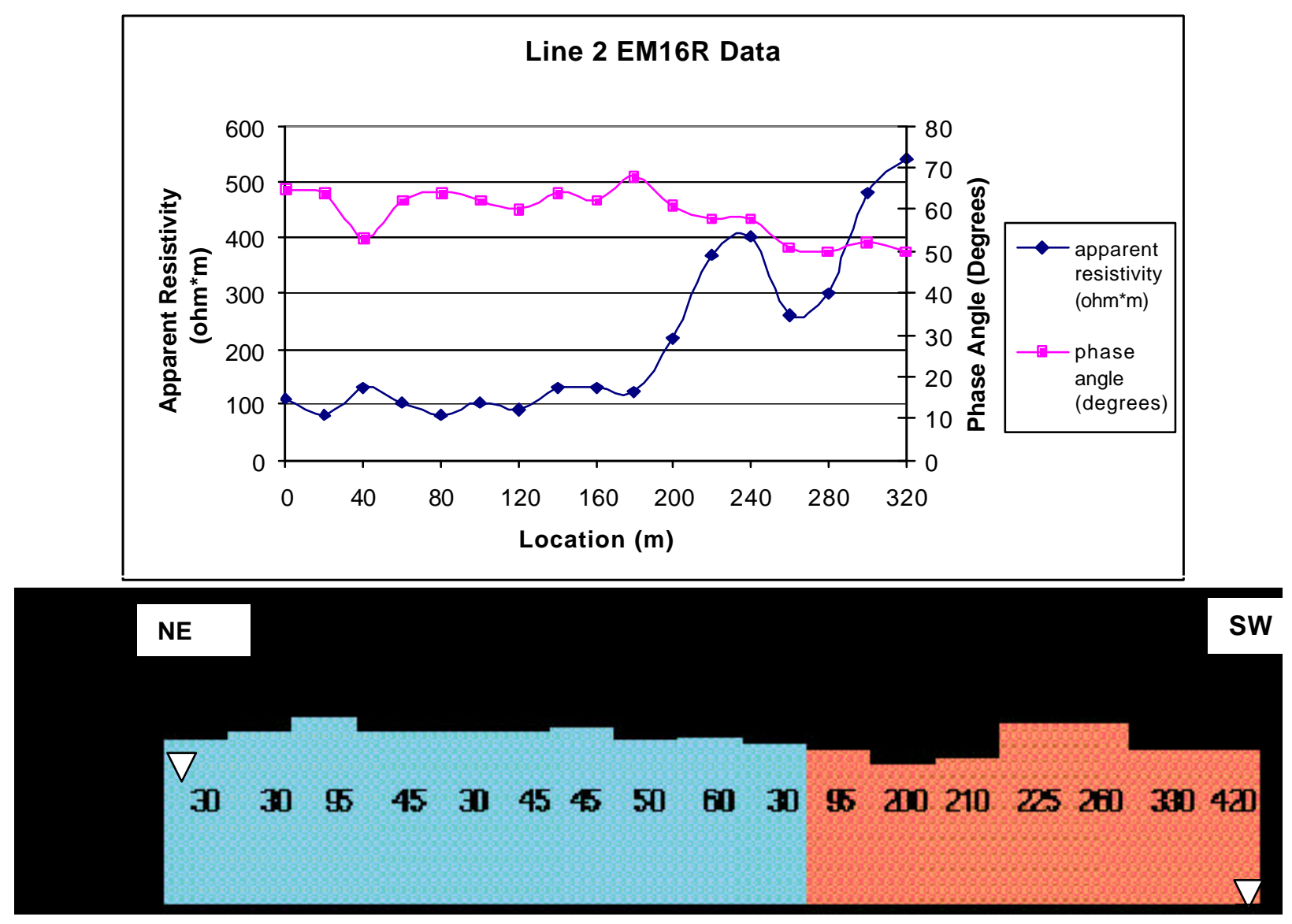

Figure 5: Line 2 VLF resistivity data and model. a. (top) Field data of apparent resistivity and impedance phase. b. (bottom) layered model assuming a first layer resistivity of $1000 \mathrm{ohm}$ meters and the second layer resistivity noted on the figure.

The VLF resistivity data (Figure 5 part a) shows common trends for both lines. The phase angle is always greater than 45 degrees and is greater at the north end of both lines (where it is 65-70 degrees), indicating an decrease in resistivity with depth. The apparent resistivity is greater on the south end of the surveys. The VLF resistivity two-layer models supports the other data sets.

There is a low Layer 2 resistivity (less than $100 \Omega$-m) from 200 meters to 350 meters, and there is a higher Layer 2 resistivity (greater than $150 \Omega-\mathrm{m}$ ) from 375 to 600 , as indicate by the orange color. Also, the thickness of Layer 1 is relatively small from 50 to 100 meters but increases to a maximum near 320 meters. Based on the change in the modeled resistivity in Layer 2 and the pattern in the thickness of Layer 1, the change in elevation of the water table is near 200 meters. The figure shows a large lateral change in Layer 2 resistivity. From 0 to 200 meters the resistivity is below $100 \Omega$-m and from 220 to 320 meters, the resistivity is greater than $200 \Omega$-m. The thickness of Layer 1 one does not show a dramatic of a change from one end of the line to the other. Based on layer 2 resistivity, the groundwater table is shallower from 
distances 0 to 200 meters and deeper from distances 220 to 320 meters, and the fault would most likely be located roughly in the 200-220 meter range.

In comparison to the models obtained with the other methods, it is clear that the VLF resistivity clearly detects the fault and the transition to a deeper water table at the southwest end of the line. The second layer detected by VLF resistivity is a composite response of the vadose and saturated zones.

\section{Conclusions}

The hydrogeology of the Desert Hot Springs area is made complex by the presence of at least one fault, which acts as a barrier to groundwater flow. A better understanding of what causes these faults to be barriers will enhance our knowledge of the flow of water through the fault. Results from resistivity soundings and HLEM, VLF and VLF-resistivity surveys support the notion that the groundwater is higher on the north end of the survey lines and then drops significantly when the southwest block of the fault is crossed. HLEM does not appear to do a good job imaging the fault zone or give a good indication of the nature of the fault (conductive or resistive nature). The equivalent current density interpretation of Line 2 data shows that there is a concentration of current near station 160 that which we interpret as the water table rising near the fault or the edge effect of the shallower conductor to the north east. The VLF resistivity data indicates a step change in apparent resistivity at about 200 meters along Line 2, which agrees with the step change in the water table. The ease of using the equipment varies from extremely slow and cumbersome (dc resistivity) to very lightweight and convenient (VLF), all provided useful information. The more cumbersome methods provided greater depth of penetration and more detail about the subsurface.

\section{Acknowledgements}

Michigan Technological University supported the field program. Undergraduate students Melissa $\mathrm{Li}$ and Amber Harris assisted in with the field measurements.

\section{References}

1. Proctor, R. J., 1968, Geology of the Desert Hot Springs-Upper Coachella Valley Area, California. Special Report 94 California Division of Mines and Geology, San Francisco, 50 p.

2. May, Wesley Lewis, 1996, Characterization of a large fault zone as a barrier to fluid flow: the San Andreas Fault near Desert Hot Springs, Riverside County, California, 1996.

3. Lukkarila, C., 1999, Refinement of a groundwater flow model: Upper Coachella Valley, Riverside County, California, MS Thesis, Dept of Geological Engineering and Sciences, Michigan Technological University. 
4. Zhody, A. R. and Bisdorf, R. J., 1989, Programs for the automatic processing and interpretation of Schlumberger Sounding Curves in QuickBasic 4.0, (USGS Open File Report 89-137 A)

5. McNeil, D., 1980, Terrain conductivity at low induction numbers, Technical Report TN-6, Geonics Ltd. , 1745 Meyerside Dr., Unit 8, Mississauga, Ontario, Canada L5T 1C5.

6. Interpex, 1988, EMIX-MM User's Manual, Interpex Ltd., Golden, Colorado.

7. Karous, M., Hjelt, S. E., 1983, Linear filtering of VLF dip-angle measurements, Geophysical prospecting, 31, 782-794.

8. Geonics, 1979, Operating Manual for EM 16R, Geonics Ltd., 1745 Meyerside Dr., Unit 8, Mississauga, Ontario, Canada L5T 1C5. 